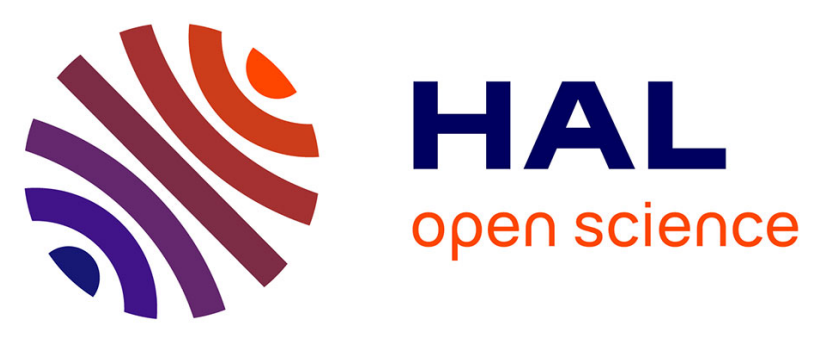

\title{
Efficacité de la réduction et fixation percutanée des fractures isolées du sacrum. Résultats fonctionnels et équilibre sagittal d'une série de 20 patients à 3,6 années de recul
}

Sébastien Ruatti, Mehdi Boudissa, Gael Kerschbaumer, Michel Milaire, Jérôme Tonetti

\section{To cite this version:}

Sébastien Ruatti, Mehdi Boudissa, Gael Kerschbaumer, Michel Milaire, Jérôme Tonetti. Efficacité de la réduction et fixation percutanée des fractures isolées du sacrum. Résultats fonctionnels et équilibre sagittal d'une série de 20 patients à 3,6 années de recul. Revue de Chirurgie Orthopédique et Traumatologique, 2019, 105, pp.481 - 486. 10.1016/j.rcot.2019.04.014 . hal-03486573

\section{HAL Id: hal-03486573 \\ https://hal.science/hal-03486573}

Submitted on 21 Dec 2021

HAL is a multi-disciplinary open access archive for the deposit and dissemination of scientific research documents, whether they are published or not. The documents may come from teaching and research institutions in France or abroad, or from public or private research centers.
L'archive ouverte pluridisciplinaire HAL, est destinée au dépôt et à la diffusion de documents scientifiques de niveau recherche, publiés ou non, émanant des établissements d'enseignement et de recherche français ou étrangers, des laboratoires publics ou privés.

\section{(ㄷ)(1) $\$$}

Distributed under a Creative Commons Attribution - NonCommerciall 4.0 International 
Version of Record: https://www.sciencedirect.com/science/article/pii/S1877051719301327

Manuscript_8188b52ae106093107e00d528a68a070

Original article

Efficacité de la réduction et fixation percutanée des fractures isolées du sacrum. Résultats fonctionnels et équilibre sagittal d'une série de 20 patients à 3.6 années de recul

Effectiveness of Closed Reduction and Percutaneous Fixation of Isolated Sacral

Fractures. Functional Outcomes and Sagittal Alignment after 3.6 Years in 20 Patients

Sébastien Ruatti; Mehdi Boudissa; Gael Kerschbaumer; Michel Milaire; Jérôme Tonetti

Chu Grenoble, France

Auteur correspondant: Dr. Sébastien Ruatti, MD

CHU Grenoble

Avenue des Maquis du Grésivaudan

38700La Tronche, FRANCE

sruatti@chu-grenoble.fr

Ne pas utiliser, pour citation, la référence française de cet article, mais celle de l'article original paru dans Orthopaedics \&Traumatology: Surgery \& Research, en utilisant le DOI ci-dessus.

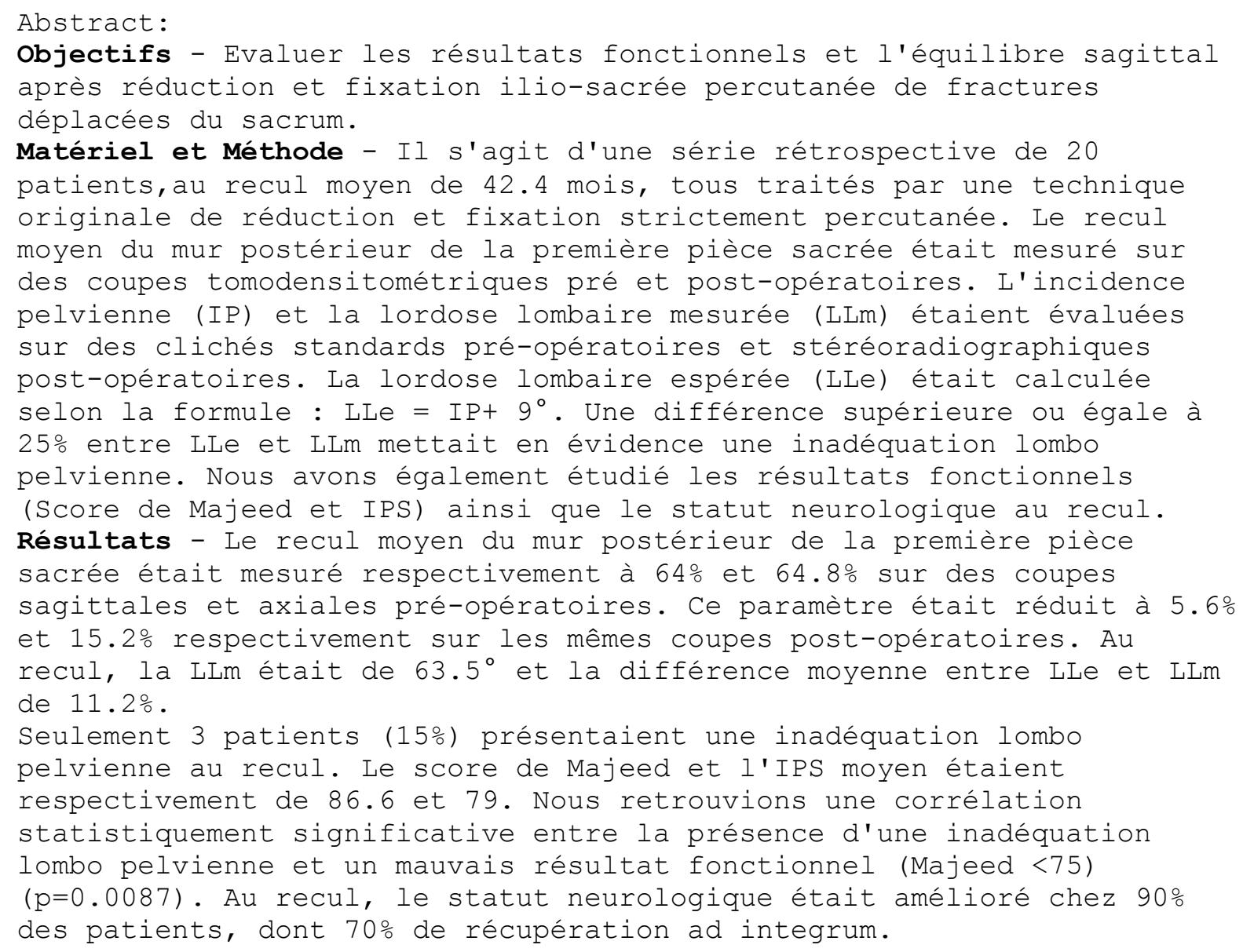




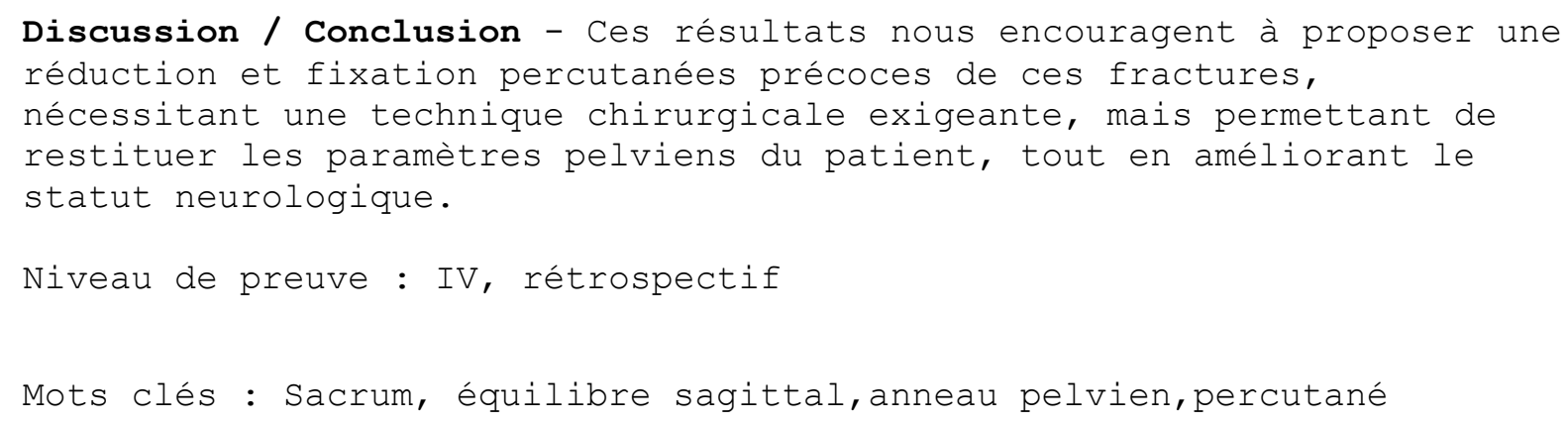

\section{Introduction}

Si 15 à 45\% des ruptures de l’anneau pelvien postérieur impliquent le sacrum [1, 2], seulement 2 à $5 \%$ sont des fractures isolées en U ou en H [3, 4]. Depuis 1985, les travaux de Roy Camille ont aidé à comprendre les mécanismes physio pathologiques de ce type de lésion, qui survient classiquement lors de la réception d'une chute d'un lieu élevé (défenestration volontaire), impliquant hyperlordose et extension des hanches [5]. Roy Camille fut le premier à décrire une classification à 3 stades et nous devons à Strange-Vognsen la description des fractures comminutives du corps de la première vertèbre sacrée (Grade 4) [6].

Pour certains auteurs, le traitement chirurgical par fixation lombo pelvienne ou trans sacrée n'a pas montré les preuves de son efficacité, en comparaison avec le traitement orthopédique ou fonctionnel [7-12]. Néanmoins, les patients présentant une inadéquation lombo pelvienne dû à une fracture déplacée peuvent déclencher des lombalgies chroniques [13]. Les méta-analyses récentes confirment les bénéfices du traitement chirurgical, grâce à la restitution des paramètres pelviens physiologiques $[12,13]$, mais au prix du risque de complications cutanées et infectieuses des chirurgies à ciel ouvert [14-17]. 
Les vissages ilio sacro iliaques percutanés, popularisés par Routt en 1996, ont permis de diminuer la morbidité du traitement chirurgical dans ce type d'indication [18, 19].

Depuis 2011, nous avons pour habitude de traiter ces fractures déplacées (Roy Camille Grade II ou III) avec une technique de réduction précoce associant tractions trans condyliennes lourdes (15\% du poids du corps) et contre traction manuelle puissante. La réduction est alors complétée par la mise en place d'un billot (fait de draps roulés) placé sous la jonction lombo sacrée. La fixation définitive est assurée par un vissage ilio sacré percutané [20] (Figure 1).

Le but de notre étude était d'évaluer les résultats d'une série continue de patients présentant une fracture en $\mathrm{U}$ ou $\mathrm{H}$ du sacrum, traités par cette technique de réduction et fixation percutanée dans un Trauma Center Universitaire de Niveau 1. Deux hypothèses de travail ont été formulées. Premièrement, la technique de réduction et fixation pourrait permettre d'obtenir une réduction anatomique, corrigeant les déséquilibres sagittaux due à la cyphose intra sacrée. Deuxièmement, la réduction anatomique pourrait éviter le recours à une laminectomie de décompression, tout en améliorant le statut neurologique des patients.

\section{Matériel et Méthode}

Entre Septembre 2007 et Juillet 2016, nous avons inclus 20 patients, 10 femmes et 10 hommes. Au moment de la prise en charge chirurgicale, l'âge moyen était de $36.5 \pm$ 11.1 ans (17-69). Tous les patients ont accepté le recueil de leurs données, l'évaluation fonctionnelle portait donc sur les 20 patients. 


\section{Critères d'inclusion}

Les critères d'inclusion était l'existence d'une fracture déplacée en $\mathrm{U}$ ou $\mathrm{H}$ du sacrum (Roy Camille grades II ou III) et un patient non décédé au recul.

\section{Techniques chirurgicales}

Cinq chirurgiens rompus aux chirurgies de l'anneau pelvien ont réalisé les gestes chirurgicaux. Un amplificateur de brillance 2D a été utilisé pour toutes les procédures. La technique de réduction précoce $(<24$ heures) et de fixation définitive percutanée a été bien décrite et publiée par notre équipe chirurgicale [20] (Figure 1).

A la phase précoce, en salle opératoire, une traction trans condylienne bilatérale était mise en place. Le patient était placé en décubitus dorsal sur la table opératoire en position de Trendelenburg. Après sédation et relâchement musculaire, le chirurgien appliquait une traction forte et explosive sur les 2 tractions, assisté par 2 aides qui réalisaient une contre traction manuelle sous les aisselles. Idéalement, la perception d'un craquement signait la désimpaction du foyer de fracture sacré. La réduction était maintenue par une traction bilatérale lourde à $15 \%$ du poids du corps. Selon les principes du Damage Control Orthopédique, il était alors possible d'attendre le moment opportun pour assurer la fixation percutanée définitive, le patient étant placé en soins de réanimation avec les tractions en place, si son état était trop précaire.

Quand l'état général était stabilisé, la fixation définitive pouvait être réalisée. Le patient était placé en décubitus dorsal sur une table radio transparente avec les 2 
tractions trans condyliennes en place. Un billot rigide et volumineux (fait de draps roulés) était placé sous la charnière lombo sacrée pour accentuer l’hyperlordose, complétant ainsi la réduction de la cyphose intra sacrée. La qualité de la réduction était appréciée sur des clichés fluoroscopiques de profil. Pour nous, l’un des meilleurs critères de réduction était la restitution de l'alignement de la corticale sacrée antérieure.

Dans tous nos cas, la fixation a été bilatérale au moyen de vis titane canulées de $7.3 \mathrm{~mm}$. Nous avons implanté 4 vis ( 2 de chaque côté) dans 6 cas, 3 vis ( 2 vis d'un côté et une de l'autre) dans 3 cas et 2 vis (une de chaque côté) dans 11 cas. Le nombre de vis implantées dépendait surtout de la taille de l'espace disponible entre le $1^{\text {er }}$ foramen sacré et le plateau supérieur de la 1ère pièce sacrée, ainsi que des sensations du chirurgien. L'autre critère était l'appréciation de la qualité osseuse du patient par le chirurgien (plus l'os est fragile, plus le nombre de vis était important).

\section{Recommandations post-opératoires}

La remise en charge était autorisée entre 15 et 45 jours post-opératoires, dépendant du chirurgien et de la qualité osseuse.

\section{Classification radiologique}

La classification radiologique était basée sur les travaux de Roy Camille et Strange-Vognsen $[5,6]$.

Le bilan d'imagerie pré et post-opératoire comprenait une radiographie du bassin et du sacrum de profil avec mesure de l'incidence pelvienne pré opératoire. Il était 
également réalisé des coupes tomodensitométriques sagittales et axiales afin de mesurer le recul du mur postérieur au niveau S1. Ces clichés permettaient également de classer la fracture selon Roy Camille.

La consolidation osseuse était appréciée sur des clichées radiographiques standards (face + profil) systématiques 6 mois après la chirurgie. Au recul, des clichés stéréoradiographiques sur machine EOS ont été réalisées afin de mesurer l'équilibre sagittal (incidence pelvienne et lordose lombaire) et évaluer notre 1 ère hypothèse. Ces données ont été appréciées par les auteurs et par un radiologue indépendant entrainé.

En post-opératoire, l'incidence pelvienne (IP) et la lordose lombaire mesurée (LLm) ont été colligées sur des clichés stéréoradiographiques EOS de profil. La lordose lombaire espérée (LLe) était calculée à l’aide de la formule LLe = IP + 9 . LLe et LLm était alors comparée. Une différence supérieure ou égale à 25\% définissait l'existence d'une inadéquation lombo pelvienne (lumbo pelvic mismatch (LPM)) [13].

\section{Evaluation fonctionnelle}

Pour chaque patient, nous avons évalué l'importance des douleurs (Echelle visuelle analogique EVA) ainsi que les résultats subjectifs mesurés avec les scores de Majeed et IPS. Les patients étaient convoqués à 6 semaines, 3, 6 et 12 mois postopératoires. Lorsque le résultat fonctionnel était mauvais (défini selon nous par un score de Majeed <75 au recul), nous avons recherché une corrélation avec la présence d'une inadéquation lombo pelvienne (lumbo pelvic mismatch (LPM)). Les données ont été analysées grâce à un test de Fisher.

Au recul, le statut neurologique était également recueilli et comparé au statut pré opératoire, afin d'évaluer notre seconde hypothèse. 


\section{Résultats}

\section{Mécanismes lésionnels}

Nous retrouvions 3 accidents de parapente, 2 chutes de cheval, une chute d'échafaudage de 3mètres, 5 défenestrations volontaires (de 3 à 12 mètres) , un accident de speedriding, un accident de moto, 3 accidents de voiture, 2 chutes de 2 et 3 mètres en ski alpin, une chute de $3 \mathrm{~m}$ en snowboard et une chute de la hauteur (Tableau 1).

\section{Evaluation radiographique}

Toutes les fractures étaient classées Grade 2 selon la classification de Roy Camille modifiée Strange-Vognsen. Nous retrouvions 10 fractures en U, 9 fractures en H et 1 fracture en $Y$.

Sur les radiographies pré opératoires du sacrum en vue de profil, l’incidence pelvienne moyenne (IP pré) était de $61.42^{\circ}$ (53.5-70). Sur les clichés tomodensitométriques pré opératoires, le recul moyen du mur postérieur au niveau S1 était respectivement de 64 et $64.8 \%$ sur des coupes sagittales et des coupes axiales. En post-opératoire immédiat, ce paramètre était respectivement réduit en moyenne à 5.6 et 15.2\% sur les coupes sagittales et axiales (Figures 2 à 5). 
Au recul moyen de 42.4 mois (12-121), les paramètres pelviens et l'équilibre sagittal étaient mesurés par un radiologue indépendant et entrainé, sur des clichés stéréoradiographiques EOS. L’incidence pelvienne post-opératoire moyenne (IP post) était de $49.25^{\circ}$ (41-56). En moyenne, la lordose lombaire mesurée (LLm), entre le plateau vertébral supérieur de L1 et le plateau vertébral inférieur de L5, était de $57.8^{\circ}$ (51.2-70.4). Grâce à la formule LLe = IP + 9º, la lordose lombaire moyenne espérée était calculée à $63.5^{\circ}$ (50-65). Ainsi la différence moyenne entre LLe et LLm était de 11.2\%. Seulement 3 patients (15\%) présentaient une inadéquation lombo pelvienne au recul, définie par une différence supérieure ou égale à 25\% entre LLe et LLm selon BoyoudGarnier et al. (Tableau 1).

\section{Complications}

Nous n'avons noté aucune complication cutanée ou infectieuse, du fait du caractère strictement percutané de la technique de réduction et de fixation.

Cependant, nous avons colligé 2 complications neurologiques iatrogènes avec une vis ilio sacrée rentrant en conflit avec une racine S1. L'ablation de la vis fut nécessaire à 6mois post-opératoire et la récupération neurologique fut complète.

\section{Résultats fonctionnels}

Tous les patients inclus ont pu être revus au recul moyen de 42.4 mois (12-121). Dix-neuf ont été opéré en 1 temps, et seulement un a du bénéficié d'une fixation définitive 8 jours après la manœuvre de réduction, du fait de la précarité de son état général. 
Le délai moyen entre la survenue du traumatisme et la chirurgie était de 1.4 jours 0-8). La remise en charge a été autorisée entre 15 et 45 jours post-opératoires, au vu de la qualité osseuse et de la complexité de la fracture. En moyenne la remise en charge a été obtenue 37 jours après la chirurgie.

Les résultats subjectifs retrouvaient 10 excellents, 9 très bons et 1 bon résultat. L'échelle visuelle analogique moyenne était de $0.87 / 10$. Les scores fonctionnels moyens de Majeed et IPS étaient respectivement de 86.6 et 79.

Dans le but de confronter notre 1 ère hypothèse, à savoir la corrélation entre la réduction anatomique et le bon résultat fonctionnel, nous avons comparé la lordose lombaire espérée (LLe) et la lordose lombaire mesurée (LLm), en accord avec les travaux de Boyoud-Garnier et al. Grâce à un test de Fisher, nous retrouvions une corrélation significative entre la présence d'une inadéquation lombo pelvienne et un faible score fonctionnel (Majeed $<75)(p=0.0087$, Cov 0.58).

Ainsi 94\% des patients avaient repris leur activité professionnelle au recul.

\section{Statut neurologique}

Le statut neurologique pré opératoire des patients était le suivant : 8 paresthésies unilatérales dans le territoire S1, 3 syndromes de la queue de cheval associé une paralysie du tronc lombo sacré dans 1 cas, 1 paralysie du tronc lombo sacré due à une compression de 2 heures sur l'hémi bassin homolatéral et 1 radiculalgie S1 bilatérale. Sept patients ne présentaient aucun signe neurologique à l'arrivée.

Au recul, nous avons colligé 90\% d'amélioration neurologique, au moins partielle. Soixante-dix pourcents des patients avaient une récupération neurologique ad integrum. Tous les statuts neurologiques sont présentés dans le Tableau 1. 


\section{Lésions associées}

La revue des lésions associées retrouvait 1 patient ayant présenté une fracture du tibia et du fémur, 1 patient avec une disjonction symphysaire en choc hémorragique, et 2 patients avec une fracture des 2 cadres obturateurs.

\section{Discussion}

Dans le cadre du traitement des fractures isolées du sacrum, certains auteurs ont la préférence du traitement conservateur, qui peut être entrepris dans des structures hospitalières n'ayant pas l'habitude de ces chirurgies. En effet, bien conduit, le traitement orthopédique reste un meilleur choix pour le patient qu'une chirurgie hasardeuse et non maitrisée [20-22], particulièrement sur des patients polytraumatisés, ce qui est souvent le cas dans ce genre de traumatisme.

Toutefois, pour la majorité des auteurs, le traitement est unanyme : réduction et fixation à ciel ouvert [14-16, 23-31]. Les complications principales de ces abords étendus restent l'infection du site opératoire et les complications cutanées, avec un taux moyen de $16 \%$ selon Bellabarba $[23,24]$.

En 1993, Pohlemann attirait déjà notre attention sur les risques infectieux que font courir ces chirurgies à ciel ouvert, et également sur la complexité des montages de 
fixation, réservés à des équipes expérimentées [32]. Pour diminuer les risques de complications, les techniques de vissage ilio sacré percutané ont progressivement vues le jour. Elles permettent de conserver l'hématome pelvien fracturaire et de diminuer les pertes sanguines per et post opératoires $[18,19]$. La technique décrite dans cette étude trouve sa place dans le domaine des chirurgies percutanées, et la réduction initiale peut être pratiquée précocement en salle d'arrivée. Ce geste nous est apparu d'autant plus efficace qu'il est réalisé précocement lors de la prise en charge du patient, car la réduction est facilitée par la mobilité fracturaire. Dans notre expérience, passée 72 heures, la réduction est rendue beaucoup plus incertaine. Ainsi dans notre série, le délai moyen entre l'accident et la réduction (associée à une fixation d'emblée dans 19 cas sur 20) était de 1.4 jours, témoignant de l'engagement de nos équipes anesthésiochirurgicales à réduire ces fractures au plus vite. La fixation définitive peut être réalisée dans le même temps chirurgical, ou différée, selon les principes du Damage Control Orthopédique. La puissance de la technique de réduction, associée à l'effet mécanique du billot lombaire créant une hyperlordose, permet de réduire la bascule postérieure de la 1ère pièce sacrée, diminuant alors dans le même temps la perturbation de l'équilibre sagittal [33]. Cette analyse se rapproche de la technique récemment décrite par Irifune et al. [33] à la différence qu'aucune traction trans osseuse n'est appliquée. En effet selon Irifune et al. [33], la seule application d'une hyperlordose forcée, contrôlée sous amplification de brillance suffirait à restituer les paramètres pelviens physiologiques du patient, avec comme mesure principale la réduction de cyphose intra sacrée liée à la fracture. Selon nous, une traction importante selon la technique décrite précédemment [20] permet de parfaire la réduction grâce au ligamentotaxis, tout en évitant de forcer sur la charnière sacrée antérieure créée par la fracture. Nous pensons que l'accrochage 
des 2 corticales sacrées antérieures est un bon élément de stabilité réductionnelle, en complément de la fixation percutanée, en créant un phénomène d'engrènement fracturaire.

Dans notre série, seulement 15\% des patients (3) présentaient une dissociation lombo pelvienne selon Boyoud-Garnier et al. [13] et les résultats subjectifs apparaissaient globalement très bons (10 excellents, 9 très bons et 1 bon résultats) : 94\% des patients avaient repris leur activité professionnelle. Ainsi nous avons trouvé une forte corrélation entre la présence d'une dissociation lombo pelvienne et un faible résultat fonctionnel (Majeed $<75)(\mathrm{p}=0.0087$, Cov 0.58), encourageant la restitution des paramètres pelviens physiologiques du patient. C'est pourquoi nous partageons l'opinion de Hart et al., pour lequel la restitution des paramètres pelviens physiologiques du patient permettrait d'anticiper la qualité de la récupération fonctionnelle [12]. De plus, Boyoud-Garnier et al. retrouvait également une forte corrélation entre la présence d'une lombalgie chronique et une perturbation des paramètres pelviens [13]. Dans notre série, nous n’avons pas noté de déplacement secondaire ni de perte de réduction au recul, si bien que la présence d'une inadéquation lombo pelvienne était uniquement due à une insuffisance de réduction initiale.

Cette notion semble être commune avec l'analyse du déplacement du mur postérieur de S1. En effet dans notre travail, plus le déplacement du mur postérieur est important, plus le bénéfice de la technique de réduction l'est aussi. Ainsi, pour les patients dont les fractures étaient les plus déplacées sur les coupes sagittales pré opératoires ( $>50 \%$ de recul du mur postérieur de S1), la technique de réduction a permis de réduire le recul du mur postérieur jusqu'à 5.6\% en moyenne en post 
opératoire. Ce paramètre n'atteignait alors que $11 \%$ chez les patients dont les fractures étaient les moins déplacées ( $<50 \%$ de recul du mur postérieur de S1) ( $p=0.008)$.

Les méta-analyses récentes [16, 25] semblent confirmer les bénéfices de la laminectomie, pour libérer les racines nerveuses étirées ou contuses. En effet, les racines avulsées n'ont que de faible chance de récupération. Selon Yi et al., la laminectomie doit être réalisée dans le même temps chirurgical et seulement avant la réduction et la fixation définitive [16]. Dans cette métaanalyse, il apparaît en effet que l'expulsion de petits fragments osseux libres vers les racines nerveuses piégées dans le canal sacré est un risque important lors des manœuvres de réduction et de fixation. Dans notre expérience, la qualité de la réduction percutanée nous est apparue suffisante pour décomprimer indirectement les racines nerveuses (Figures 2 à 5). Nous avons ainsi évité la morbidité due aux complications cutanées et infectieuses des chirurgies à ciel ouvert [24]. Nos résultats restent néanmoins très bons comparés à la littérature avec $90 \%$ de récupération neurologique, dont 70\% de récupération ad integrum. George retrouvait $16 \%$ de récupération quasi complète et $67 \%$ d'amélioration partielle [26], là où Schildhauer et Bellabarba retrouvaient $83 \%$ de récupération neurologique, partielle ou complète, toujours après laminectomie et fixation interne [24]. Dussa retrouvait 40\% d'amélioration neurologique grâce à la réduction et fixation à ciel ouvert [15], là où Fountain présentait $100 \%$ de récupération neurologique après laminectomie sur des tableaux de rétention aigue d'urine [29].

Dans notre service, nous réservons la laminectomie sacrée aux rares cas où le patient ne présente pas d'amélioration neurologique après la réduction et fixation percutanée, et seulement pour extraire des fragments osseux très déplacés projetés 
dans le foramen S1 ou le canal sacré. Nous n'y avons cependant jamais eu recours dans la série présentée.

Les limites de notre étude sont le caractère rétrospectif des inclusions, ainsi que le faible nombre de patients, en dépit de la rareté de cette fracture isolée.

\section{Conclusion}

Les fractures isolées du sacrum sont des lésions graves, devant être prises en charge dans des Trauma Center de Niveau 1, rompus à la chirurgie traumatique du rachis et de l'anneau pelvien. Les fractures déplacées nécessitent toujours, dans notre expérience, une intervention chirurgicale. Basée sur l’expérience de cette série de 20 patients, il nous apparaît que l'association d'une réduction précoce et d'une fixation percutanée est une méthode fiable pour restaurer les paramètres pelviens physiologiques du patient. Notre $1^{\text {re }}$ hypothèse est validée : cette technique de réduction et fixation percutanée permet d'obtenir une réduction anatomique dans la plupart des cas, corrigeant la modification des paramètres pelviens par la cyphose traumatique intra sacrée.

Cette technique permet également d'améliorer le statut neurologique dans 90\% des cas, validant notre 2ème hypothèse de travail : la réduction anatomique permet d'éviter la laminectomie de décompression. L’incidence des complications cutanées et infectieuses des chirurgies à ciel ouvert s'en trouve considérablement diminuée, permettant une mobilisation précoce et diminuant la morbi mortalité de ce type de lésion. 


\section{Conflits d'intérêt :}

Chaque auteur certifie qu'il n'a pas d'association commerciale qui pose un conflit d'intérêt avec cet article.

Sources de financement : aucune

\section{Contribution des auteurs :}

Le Dr RUATTI a rédigé l'article et colligé les données.

Les Dr BOUDISSA, KERSCHBAUMER et MILAIRE ont colligé des données et aidé à la construction de la trame de rédaction.

Le Pr TONETTI a instauré la dynamique de l'étude et participé à la rédaction de l'article. L'ensemble des auteurs a contribué à opérer les patients. 


\section{REFERENCES}

1. Denis F, Davis S, Comfort T. Sacral fractures: an important problem : retrospective analysis of 236 cases. Clin Orthop. 1988;227:67-81

2. Taguchi T, Kawai S, Kaneko K et al. . Operative management of displaced fractures of the sacrum. J Orthop Sci. 1999;4:347-52.

3. Carl A, Delman A, Engler G. Displaced transverse sacral fractures: a case report, review of the literature, and the CT scan as an aid in management. Clin Orthop. 1985;194:195-8

4. Phelan ST, Jones DA, Bishay M. Conservative management of transverse fractures of the sacrum with neurological features: a report of four cases. J Bone Joint Surg Br. 1991;73:969-71

5. Roy-Camille R, Saillant G, Gagna G et al. Transverse fracture of the upper sacrum. Suicidal jumper's fracture. Spine (Phila Pa 1976). 1985;10:838-45

6. Strange-Vognsen $\mathrm{HH}$, Lebech A. An unusual type of fracture in the upper sacrum. J Orthop Trauma. 1991;5:200-3

7. Wiesel SW, Zeide MS, Terry RL. Longitudinal fractures of the sacrum: case report.J Trauma. 1979;19:70-1

8. Ferris B, Hutton P. Anteriorly displaced transverse fracture of the sacrum at the level of the sacro-iliac joint. A report of two cases. J Bone Joint Surg Am. 1983;65:407-9

9. Dogra AS, Karkhanis AR, Asurlekar RV. Fracture sacrum. J Postgrad Med. 1995 ;41:47- 
10. Ikada K, Wada E, Kodama N. Traction injury of the lumbosacral spinal nerve roots: report of a case. Spine. 1991;16:368-71

11. Mouhsine E, Wettstein M, Schizas C et al. Modified triangular posterior osteosynthesis of unstable sacrum fracture. Eur Spine J. 2006;15:857-63.

12. Hart RA, Badra MI, Madala A. Use of pelvic incidence as a guide to reduction of $\mathrm{H}-$ type spino-pelvic dissociation injuries. J Orthop Trauma 2007;21:369-74

13. Boyoud-Garnier L, Boudissa M, Ruatti S et al. Chronic low back pain after lumbosacral fracture due to sagittal and frontal vertebral imbalance. Orthop Traumatol Surg Res. 2017 Jun;103(4):523-526. doi: 10.1016/j.otsr.2017.01.013.

14. Robles LA. Transverse sacral fractures. Spine J. 2009;9:60-9.

15. Dussa CU, Soni BM. Influence of type of management of transverse sacral fractures on neurological outcome. A case series and review of literature. Spinal Cord. $2008 ; 46590-4$

16. Yi C, Hak DJ. Traumatic spinopelvic dissociation or U-shaped sacral fracture: a review of the literature. Injury. 2012;43402-8.

17. Hak DJ, Baran S, Stahel P. Sacral fractures: current strategies in diagnosis and management. Orthopedics. 2009;32(10). doi: 10.3928/01477447-20090818-18.

18. Routt ML Jr, Simonian PT. Closed reduction and percutaneous skeletal fixation of sacral fractures. Clin Orthop Relat Res. 1996;329:121-8.

19. Routt ML Jr, Nork SE, Mills WJ. Percutaneous fixation of pelvic ring disruptions. Clin 
Orthop Relat Res. 2000;375:15-29

20. Ruatti S, Kerschbaumer G, Gay E et al. Technique for reduction and percutaneous fixation of U- and H-shaped sacral fractures.

Orthop Traumatol Surg Res. 2013;99(5):625-9. doi: 10.1016/j.otsr.2013.03.025.

21. Schwab F, Lafage V, Patel A et al. Sagittal plane co,siderations and the pelvis inj the adult patient. Spine (Phila Pa 1976) 2009; 34 :1828-33.

22. Kellam JF, McMurtry RY, Paley D et al. The unstable pelvic fracture. Operative treatment.Orthop Clin North Am. 1987;18(1):25-41

23. Helgeson MD, Lehman RA Jr, Cooper P et al. Retrospective review of lumbosacral dissociations in blast injuries. Spine (Phila Pa 1976). 2011;36(7):E469-75

24. Schildhauer TA, Bellabarba C, Nork SE, et al. Decompression and lumbopelvic fixation for sacral fracture-dislocations with spino-pelvic dissociation. J Orthop Trauma 2006;20:447-57.

25. Kim MY , Reidy DP , Nolan PC et al .Transverse sacral fractures: case series and literature review. Can J Surg. 2001;44(5):359-63.

26. George S. Sapkas, Andreas F. Mavrogenis et al. Transverse sacral fractures with anterior displacement. Eur Spine J. 2008; 17(3): 342-7

27. Gribnau AJ, Van Hensbroek PB, Haverlag R et al. U-shaped sacral fractures: surgical treatment and quality of life. Injury. 2009;40(10):1040-8.

28. Vresilovic EJ, Mehta S, Placide R, et al. Traumatic spondylopelvic dissociation. A report of two cases. J Bone Joint Surg Am 2005;875:1098-103 
29. Fountain SS, Hamilton RD, Jameson RM. Transverse fractures of the sacrum. A report of six cases. J Bone Joint Surg Am. 1977;59(4):486-9

30. Vaccaro AR, Kim DH, Brodke DS, et al. Diagnosis and management of sacral spine fractures. Instr Course Lect 2004;53:375-85

31. König MA, Seidel U, Heini P et al. Minimal-invasive Percutaneous Reduction and Transsacral Screw Fixation for U-shaped Fractures. J Spinal Disord Tech. 2013;26(1):4854. doi:10.1097/BSD.0b013e3182318539

32. Pohlemann T, Angst M, Schneider E et al. Fixation of transforaminal sacrum fractures: a biomechanical study J Orthop Trauma. 1993;7(2):107-17

33. Irifune H, Hirayama S, Takahashi $\mathrm{N}$ et al. Closed Reduction in a "Hyperextended Supine Position" with Percutaneous Transsacral-Transiliac and Iliosacral Screw Fixation for Denis Zone III Sacral Fractures. Adv Orthop. 2018 May 23;2018:6098510. doi: 10.1155/2018/6098510. eCollection 2018. 


\section{LEGENDES}

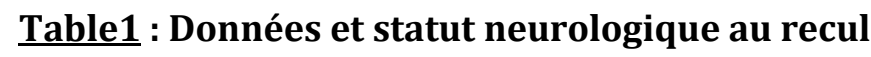

Figure 1 : Représentation de la technique de réduction précoce et installation en salle opératoire. A noter le billot lombaire, et l'amplificateur de brillance en vue de profil pour apprécier la qualité de la réduction

Figure 2 : Coupes tomodensitométriques en vue sagittale pré opératoire: recul du mur postérieur de $S 1$ et cyphose intra sacrée.

Figure 3 : Coupes tomodensitométriques en vue axiale pré opératoire: recul du mur postérieur de S1 et cyphose intra sacrée.

Figure 4 : Illustration des résultats sur coupes tomodensitométriques : Réduction du recul du mur postérieur de S1, obtenue par la technique de réduction , puis maintenue par vissage ilio sacro iliaque. Vue sagittale post opératoire

Figure 5 : Illustration des résultats sur coupes tomodensitométriques : Réduction du recul du mur postérieur de $S 1$, obtenue par la technique de réduction , puis maintenue par vissage ilio sacro iliaque. Vue axiale post opératoire 


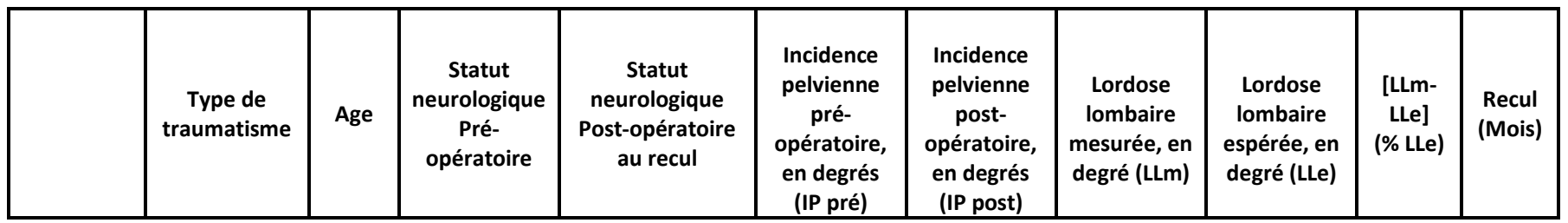




\begin{tabular}{|c|c|c|c|c|c|c|c|c|c|c|}
\hline Patient 1 & Chute en ski $2 \mathrm{~m}$ & 32 & $\begin{array}{l}\text { Dysesthésie S1 } \\
\text { gauche }\end{array}$ & $\begin{array}{l}\text { Dysesthésie S1 } \\
\text { gauche }\end{array}$ & 60 & 41 & 51.2 & 50 & 2.4 & 55 \\
\hline Patient 2 & Chute $3 \mathrm{~m}$ & 37 & $\begin{array}{c}\text { Syndrome } \\
\text { queue de } \\
\text { cheval }\end{array}$ & 0 & 66 & 43 & 58 & 52 & 11.5 & 61 \\
\hline Patient 3 & Snowboard & 40 & 0 & 0 & 64.3 & 55 & 65.1 & 64 & 12.7 & 52 \\
\hline Patient 4 & Moto & 17 & $\begin{array}{c}\text { Dysesthésie S1 } \\
\text { Bilatérale + } \\
\text { Anesthésie S2 } \\
\text { à S5 } \\
\end{array}$ & $\begin{array}{l}\text { Dysesthésie S1 } \\
\text { Bilatérale }\end{array}$ & 56.7 & 54 & 64.2 & 63 & 1.85 & 57 \\
\hline Patient 5 & Parapente & 39 & $\begin{array}{c}\text { Syndrome } \\
\text { queue de } \\
\text { cheval }+ \\
\text { paralysie } \\
\text { complète tronc } \\
\text { lombo sacré } \\
\text { droit + paralysie } \\
\text { releveurs pied } \\
1 / 5\end{array}$ & $\begin{array}{c}\text { Paralysie releveurs } \\
\text { pied } 3 / 5+\text { Paralysie } \\
\text { incomplète du tronc } \\
\text { lombo sacré }\end{array}$ & 65 & 52 & 64.5 & 61 & 5.7 & 121 \\
\hline Patient 6 & Chute de cheval & 40 & 0 & 0 & 68.6 & 50 & 62 & 59 & 5 & 51 \\
\hline Patient 7 & Parapente & 53 & $\begin{array}{c}\text { Paralysie } \\
\text { complète tronc } \\
\text { lombo sacré } \\
\text { gauche }\end{array}$ & 0 & 62.7 & 53 & 62.2 & 62 & 0.37 & 51 \\
\hline Patient 8 & Chute $7 \mathrm{~m}$ & 23 & 0 & 0 & 57.7 & 52 & 65 & 61 & 6.5 & 49 \\
\hline Patient 9 & Chute $1 \mathrm{~m}$ & 61 & 0 & 0 & 61 & 50 & 70.2 & 59 & 19 & 45 \\
\hline Patient 10 & Chute ski 3m & 22 & $\begin{array}{l}\text { Syndrome } \\
\text { queue de } \\
\text { cheval } \\
\text { incomplet }\end{array}$ & 0 & 58 & 45 & 64.8 & 54 & 20 & 44 \\
\hline Patient 11 & Parapente & 27 & 0 & 0 & 64.2 & 45 & 68.4 & 54 & 26.7 & 34 \\
\hline Patient 12 & $\begin{array}{l}\text { Accident } \\
\text { voiture }\end{array}$ & 21 & $\begin{array}{l}\text { Syndrome } \\
\text { queue de } \\
\text { cheval }\end{array}$ & 0 & 61 & 42 & 64.4 & 51 & 26.3 & 32 \\
\hline Patient 13 & Chute $9 \mathrm{~m}$ & 28 & 0 & 0 & 57 & 52 & 62.1 & 61 & 19 & 32 \\
\hline Patient 14 & Chute $5 \mathrm{~m}$ & 23 & 0 & 0 & 63 & 53 & 69 & 62 & 11.3 & 31 \\
\hline Patient 15 & Accident voiture & 23 & 0 & 0 & 61 & 51 & 64.3 & 60 & 7.2 & 53 \\
\hline Patient 16 & Accident voiture & 69 & 0 & 0 & 70 & 56 & 70.4 & 65 & 16 & 28 \\
\hline Patient 17 & Chute $12 \mathrm{~m}$ & 40 & $\begin{array}{l}\text { Dysesthésie S1 } \\
\text { gauche }\end{array}$ & 0 & 61.7 & 42 & 66.8 & 51 & 30.95 & 18 \\
\hline Patient 18 & Chute $3 \mathrm{~m}$ & 44 & 0 & 0 & 62 & 48 & 64.1 & 57 & 12.5 & 15 \\
\hline Patient 19 & Chute cheval & 34 & $\begin{array}{c}\text { Paresthésie S1 } \\
\text { Bilatérale }\end{array}$ & 0 & 53.5 & 50 & 61.4 & 59 & 4 & 14 \\
\hline Patient 20 & Speed riding & 57 & $\begin{array}{l}\text { Dysesthésie S1 } \\
\text { gauche }\end{array}$ & 0 & 55 & 51 & 53 & 52 & 1.9 & 5 \\
\hline
\end{tabular}

Tableau 1 


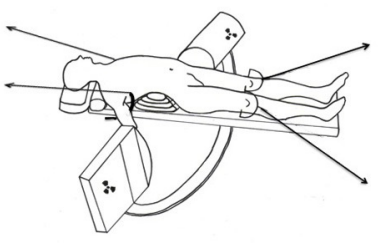




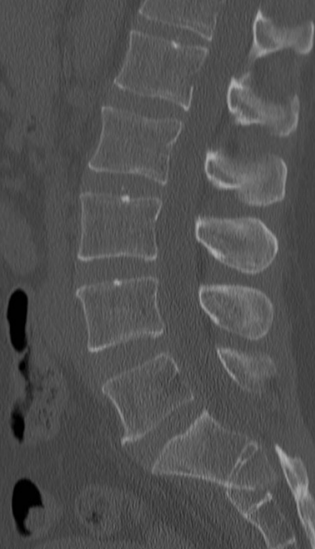




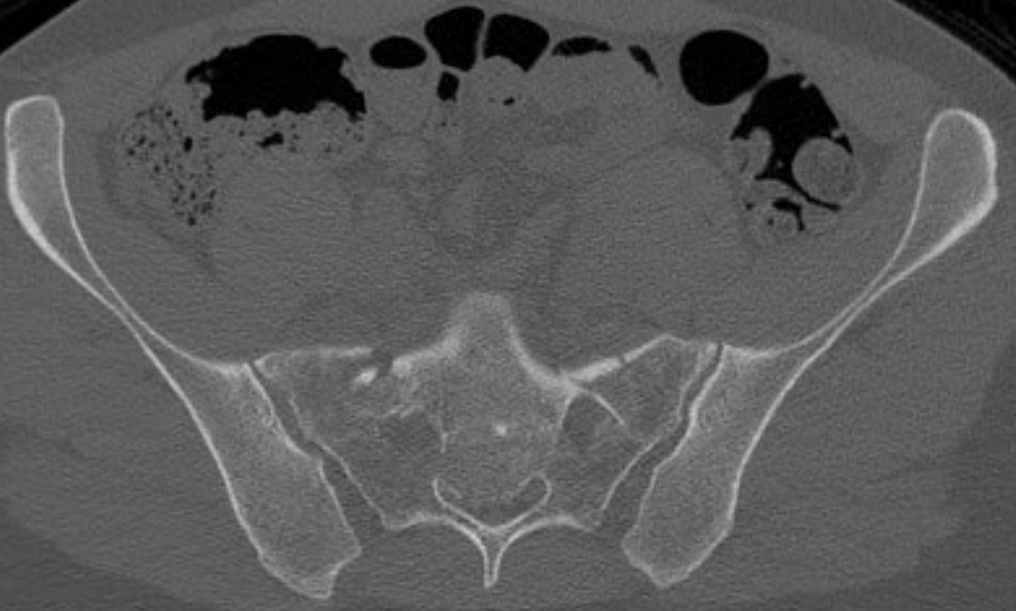



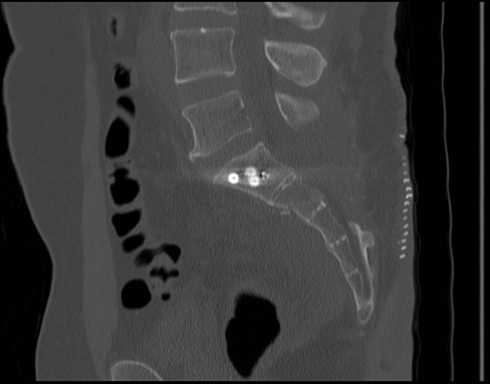
